ARTíCULO CIENTÍFICO

\title{
CALIDAD NUTRICIONAL Y CONSUMO DE FORRAJE DE MAÍZ (Zea mays) Y FORRAJE DE ESTRELLA AFRICANA (Cynodon nlemfuensis) CON O SIN ALIMENTO BALANCEADO EN CABRAS ${ }^{1}$
}

Jorge Alberto Elizondo-Salazar ${ }^{2}$

\section{RESUMEN}

El presente trabajo tuvo como objetivo evaluar la calidad y consumo del forraje de maíz y estrella africana en cabras ofrecidos de forma fresca, con o sin alimento balanceado. El experimento se llevó a cabo en la Estación Experimental Alfredo Volio Mata de la Universidad de Costa Rica. El forraje cosechado fue suministrado a 12 cabras de las razas Saanen, Toggenburg y LaMancha no lactantes y no gestantes, distribuidas en un diseño irrestricto al azar con un arreglo factorial $2 \times 2$ y con un peso vivo promedio de $57 \pm 2 \mathrm{~kg}$. Los tratamientos experimentales fueron 1) forraje de maíz, 2) forraje de maíz + alimento balanceado, 3) forraje de estrella africana y 4) forraje de estrella africana + alimento balanceado. El forraje de maíz se ofreció a los animales en forma picada y la estrella africana sin picar. Se observó un consumo promedio de materia seca de: $0,49,0,80,0,78$ y $0,93 \mathrm{~kg}$.animal ${ }^{-1} \cdot \mathrm{d}^{-1}$, para los tratamientos forraje de maíz solo, forraje de maíz + alimento balanceado, forraje de estrella africana y forraje de estrella africana + alimento balanceado, respectivamente. Esto representó 0,86; 1,$40 ; 1,37$ y $1,63 \%$ del peso vivo. El consumo de proteína cruda fue menor para el forraje de maíz sin alimento y mayor para el forraje de maíz con alimento (70,0 vs. 130,0 g.animal $\left.{ }^{-1} \cdot d^{-1}\right)$. El consumo de lignina fue en promedio de 3,5 g.animal ${ }^{-1} \cdot d^{-1}$. Este estudio demuestra que la especie de forraje influye significativamente sobre el consumo voluntario de materia seca en cabras y el contenido de materia seca en los tratamientos utilizados afectó dicho consumo.

Palabras clave: Consumo, materia seca, calidad, Capra hircus 
Nutritional quality and intake of corn fodder (Zea mays) and star grass (Cynodon nlemfuensis) with or without feed in goats. The objective of the study was to evaluate the quality and intake of corn fodder and star grass in goats offered fresh with or without concentrate. The experiment was carried out at the Alfredo Volio Mata Experiment Station of the University of Costa Rica. Forage was fed to 12 Saanen, Toggenburg and LaMancha non-lactating and non-pregnant goats distributed in a randomized $2 \times 2$ factorial design with an average live weight of $57 \pm 2 \mathrm{~kg}$. The treatments were 1) corn fodder, 2) corn fodder + feed, 3) star grass, and 4) star grass + feed. Corn fodder was chopped to the animals and star grass was not chopped. Dry matter intake was $0.49,0.80,0.78$ y $0.93 \mathrm{~kg}$. animal $^{-1} . \mathrm{d}^{-1}$, for corn fodder, corn fodder + feed, star grass, and star grass + feed, respectively. This represented 0.86, 1.40, 1.37, and $1.63 \%$ of body weight. Crude protein intake was lower for corn fodder without feed and higher for corn fodder with feed (70.0 vs. 130.0 g.animal $\left.^{-1} . \mathrm{d}^{-1}\right)$. Lignin intake was in average $3.5 \mathrm{~g}$.animal ${ }^{-1} \cdot \mathrm{d}^{-1}$. This study demonstrates that forage specie significantly affects voluntary dry matter intake in goats and that dry matter content of the treatments used in this study also affected dry matter intake.

Keywords: Intake, dry matter, quality, Capra hircus

\section{INTRODUCCIÓN}

La cabra, desde hace cientos de años, ha jugado un importante rol socio-económico en el desarrollo de la civilización alrededor del mundo. Es particularmente importante en los trópicos y subtrópicos donde es utilizada como una fuente importante de carne, leche, fibra, piel y estiércol en muchas sociedades tradicionales. También se ha utilizado como un recurso rápido de inversión (Seleka, 2001).

Su gran aptitud para la producción láctea y su facilidad de conversión alimenticia hacen que su explotación, a pequeña o gran escala, sea una alternativa para muchos productores, ya que por su pequeño tamaño y su capacidad para consumir diversas plantas disponibles, le permiten adaptarse a una gran diversidad de medios con gran facilidad. Sin embargo, no por estas razones las cabras deben ser mal alimentadas, ya 
que el desempeño de las mismas se ve afectado significativamente por una mala alimentación.

Existe en nuestro medio una serie de especies forrajeras que pueden estar disponibles en los sistemas productivos y que presentan características de calidad nutritiva, de disponibilidad, de producción de biomasa y de versatilidad agronómica importantes, representando así un excelente potencial para mejorar la calidad alimenticia e incrementar la producción de estos animales.

En este sentido, el forraje de maíz y el pasto estrella africana pueden constituirse en una alternativa viable para mejorar su alimentación.

El maíz (Zea mays), originario de México y Centroamérica, es una excelente opción forrajera que, por sus características productivas, podría ser utilizada en zonas ecológicas en donde, ni aún las especies de pastos más adaptadas, permitirán maximizar la capacidad de carga por hectárea (Fuentes et al., 2000).

El maíz pertenece a la familia Poaceae. Es un cultivo anual, con un ciclo vegetativo de 120 a 150 días para la producción de grano, dependiendo de la altitud. Se cultiva en una gran variedad de climas y es un cereal básico para la alimentación humana, constituyéndose en la base alimenticia de muchas culturas. Se cultiva con frecuencia para producir forraje verde, ya que es muy palatable y de gran valor nutritivo. Suele cosecharse cuando el grano se encuentra en estado lechoso-pastoso y las hojas están todavía verdes, obteniéndose únicamente una cosecha en cada siembra (Skerman, 1992).

Todas las variedades pueden cultivarse para forraje, pero las de mayor rendimiento son aquellas variedades regionales de porte alto. Los híbridos por su porte pequeño generalmente producen menos cantidad de forraje por unidad de área. Los rendimientos que se puedan obtener varían según la variedad, la fertilidad del suelo, la edad de corte y la densidad de siembra entre otros factores (Aldrich y Leng, 1974). Un buen cultivo de maíz forrajero puede producir entre 60 y 80 toneladas de forraje fresco por hectárea (Bernal, 1991; Skerman, 1992).

Otra especie que ha mostrado buenas características forrajeras es el pasto estrella africana (Cynodon nlemfuensis), perteneciente también a la familia Poaceae. Este pasto es una especie, que tanto por sus características de adaptación como nutricionales, es muy utilizada en los sistemas ganaderos de leche. 
Este pasto se usa ampliamente en América Latina, gracias a sus características de productividad, facilidad de establecimiento y persistencia. Se caracteriza por ser una planta perenne, con hábito de crecimiento erecto $(0,50$ a $0,75 \mathrm{~m}$ de altura) y estolonífero que cubre rápidamente el suelo. Es un forraje productivo en un amplio rango de ambientes y tipos de suelos. Se desarrolla bien en climas cálidos y secos con precipitaciones mínimas anuales de 500 a $750 \mathrm{~mm}$ y se adapta desde los cero hasta los $2000 \mathrm{msnm}$. Resiste bien los suelos con diversos grados de acidez, pero su pH óptimo se encuentra entre 5,5 y 6,0. Limita su productividad a zonas donde las temperaturas no bajen de $-5^{\circ} \mathrm{C}$. Las temperaturas altas inhiben su crecimiento, a no ser que exista un suministro adecuado de humedad y no tolera excesos de agua por periodos superiores a los 5 días. Requiere periodos de descanso que varían entre los 21 y 42 días (Elizondo, 2008).

Un principio básico en la nutrición animal es cuantificar el consumo de materia seca para determinar la ingestión de nutrientes. En ganado bovino lechero, se han llevado a cabo un número importante de ensayos para determinar el consumo de materia seca y desarrollar modelos matemáticos para predecirlo (Hristov et al., 2004). Sin embargo, en ganado caprino, el panorama es otro y el rango de valores encontrado en la literatura es muy amplio (Rodríguez y Elizondo, 2012).

En Costa Rica se han venido realizando investigaciones desde los años ochenta con diferentes tipos de forrajes como gramíneas, leguminosas, arbustivas e incluso ensilajes (Samur, 1984; Castro, 1989; Rodríguez, 1989; Vallejo, 1995; Chávez, 2005; Chacón, 2008; Herrera et al., 2009, Vargas 2009), pero los valores de consumo de materia seca obtenidos generalmente son muy bajos cuando se comparan con aquellos reportados en otras regiones del mundo. Rodríguez y Elizondo (2012) reportaron valores de consumo un poco más cercanos a los reportados por investigadores en otros países. Es por esta razón que el presente trabajo se llevó a cabo con el fin de evaluar la calidad y consumo de forraje de maíz y pasto estrella africana con o sin alimento balanceado en cabras. 


\section{MATERIALES Y MÉTODOS}

El experimento se llevó a cabo en el módulo caprino de la Estación Experimental de Ganado Lechero Alfredo Volio Mata de la Universidad de Costa Rica que se ubica a $1.542 \mathrm{msnm}$, con una precipitación media anual de $2.050 \mathrm{~mm}$, distribuidos durante los meses de mayo a noviembre con una humedad relativa media de $84 \%$ y temperatura media de $19,3 \stackrel{\circ}{ } \mathrm{C}$.

Se utilizó forraje de maíz blanco criollo forrajero que se cosechó a los 110 días de edad, y fue cortado a 0,10 m sobre el nivel del suelo. La estrella africana provino de un potrero con más de 10 años de establecida y se cortó a una edad de 45 días de rebrote.

El forraje cosechado fue suministrado a 12 cabras de las razas Saanen, Toggenburg y LaMancha no lactantes y no gestantes, con un peso vivo promedio de $57 \pm 2 \mathrm{~kg}$, distribuidas en un diseño irrestricto al azar con un arreglo factorial $2 \times 2$, donde se evaluó el tipo de forraje: maíz y estrella africana y el uso o no de alimento balanceado. Con base en lo anterior, los tratamientos experimentales fueron: 1) forraje de maíz solo, 2) forraje de maíz + alimento balanceado, 3) forraje de estrella africana y 4) forraje de estrella africana + alimento balanceado. El forraje de maíz se ofreció a los animales en forma picada $(2,5 \mathrm{~cm})$ tal como fue cosechado (tallos y hojas) el mismo día y el pasto estrella se ofreció tal como fue cosechado el mismo día, sin picar. En los tratamientos correspondientes, el alimento balanceado se mezcló con el forraje a razón de $1,0 \mathrm{~kg}$ (peso fresco) por animal por día. La composición nutricional del alimento fue de $87 \% \mathrm{MS}, 1,6 \mathrm{Mcal}_{\mathrm{EN}} \cdot \mathrm{kg} \mathrm{MS}^{-1}, 16 \% \mathrm{PC}$. Las dietas no fueron planeadas para ser isoenergéticas ni isoproteicas.

Cada animal fue ubicado en una jaula individual y recibió el tratamiento diariamente en una relación de $10 \%$ de su peso vivo como material fresco, en dos porciones iguales a las 08:00 y a las 14:00 horas.

El período experimental fue de 45 días que incluyó un periodo de adaptación de 15 días a la dieta y a las jaulas individuales, y 30 días para la medición de consumo y toma de muestras. Los animales consumieron agua ad libitum y no se suplieron sales minerales durante el ensayo. El material rechazado se recogió diariamente de los comederos individuales a las 7:30 a.m. y se pesó para evaluar el consumo, además se tomaron muestras del material ofrecido y rechazado, para analizar el contenido 
bromatológico de las dietas empleadas. Las muestras, tanto de lo ofrecido como de lo rechazado, se analizaron para determinar el contenido de materia seca, proteína cruda, fibra detergente neutro, fibra detergente ácido, cenizas, celulosa, hemicelulosa y lignina, utilizando los procedimientos aprobados (Goering y Van Soest, 1970; AOAC, 1980) en cada caso.

Para realizar los análisis de las dietas, las muestras se secaron a $65^{\circ} \mathrm{C}$ durante 48 horas y se molieron a 1 milímetro, luego se prepararon muestras compuestas semanales para el material ofrecido y el material rechazado.

Los datos obtenidos sobre consumo y composición bromatológica se analizaron mediante el PROC MIXED de SAS (SAS, 2004), donde la cabra se consideró como la variable aleatoria.

La comparación entre tratamientos se realizó utilizando la prueba de Waller-Duncan con una significancia $\alpha=0,05$.

\section{RESULTADOS Y DISCUSIÓN}

\section{Análisis de consumo}

La información sobre consumo recolectada durante el periodo experimental se sometió al análisis estadístico correspondiente y los resultados obtenidos se pueden apreciar en el Cuadro 1.

Se encontraron diferencias significativas $(P<0,05)$ en el consumo de la dieta fresca, observándose como el forraje de maíz sin alimento balanceado presentó en promedio el mayor consumo (3,71 kg.animal-1.d-1) en comparación con los demás tratamientos. Dicho valor representa un consumo del $6,51 \%$ del peso vivo. Este consumo más alto, podría ser resultado de la gran palatabilidad que presenta el forraje de maíz.

Un comportamiento similar fue reportado por Rodríguez y Elizondo (2012) quienes encontraron que el consumo de forraje fresco fue de 3,64 y 3,40 kg.animal-1.d-1 cuando los animales consumieron forraje de morera y de estrella africana, respectivamente. 
Cuadro 1. Consumo y concentración de materia seca de las dietas ofrecidas.

\begin{tabular}{|c|c|c|c|c|}
\hline \multirow[b]{2}{*}{ Dieta ofrecida } & \multicolumn{2}{|c|}{ Forraje de maíz } & \multicolumn{2}{|c|}{ Forraje de estrella africana } \\
\hline & Sin alimento & Con alimento & Sin alimento & Con alimento \\
\hline \multicolumn{5}{|l|}{ Materia fresca } \\
\hline Consumida, $\mathrm{kg} / \mathrm{d}$ & $3,71^{a}$ & $3,07^{b}$ & $2,83^{b}$ & $2,74^{b}$ \\
\hline Consumida, \%PV & $6,51^{\mathrm{a}}$ & $5,38^{b}$ & $4,96^{b}$ & $4,81^{b}$ \\
\hline \multicolumn{5}{|l|}{ Materia seca } \\
\hline Ofrecida, \% & $11,60^{d}$ & $24,46^{c}$ & $30,93^{b}$ & $41,28^{\mathrm{a}}$ \\
\hline Consumida, \% & $13,77^{\mathrm{c}}$ & $25,37^{b}$ & $27,42^{b}$ & $36,76^{a}$ \\
\hline Consumida, kg/d & $0,49^{c}$ & $0,80^{b}$ & $0,78^{b}$ & $0,93^{a}$ \\
\hline Consumida, \%PV & $0,86^{c}$ & $1,40^{\mathrm{b}}$ & $1,37^{\mathrm{b}}$ & $1,63^{\mathrm{a}}$ \\
\hline
\end{tabular}

Es importante hacer notar que el contenido de materia seca de las dietas ofrecidas presentó una diferencia estadística significativa $(P<0,05)$ entre los tratamientos utilizados. Se observaron diferencias entre especies y entre el uso o no de alimento balanceado. El pasto estrella africana con alimento balanceado presentó el mayor porcentaje de materia seca $(41,28 \%)$, mientras que el forraje de maíz sin alimento balanceado presentó la concentración más baja (11,60\%). Esta concentración de materia seca en el forraje de maíz, es muy similar a la reportada por Elizondo y Boschini (2001) quienes estudiaron el efecto de la densidad de siembra sobre el rendimiento y la calidad nutricional del forraje de maíz blanco criollo forrajero. También es muy similar a la reportada por Elizondo (2011) quien estudió la influencia de la variedad y altura de cosecha sobre el rendimiento y valor nutritivo de maíz para ensilaje.

Al analizar el consumo de materia seca $\left(\mathrm{kg}^{\mathrm{d}} \mathrm{d}^{-1}\right)$, se observó una diferencia significativa $(\mathrm{P}<0,05)$ entre los diferentes tratamientos, siendo el pasto estrella africana con alimento balanceado la dieta más consumida $(0,93 \mathrm{~kg})$, lo que representó un $1,63 \%$ del peso vivo.

El consumo de materia seca con respecto al peso vivo del animal, rondó en promedio el $1,3 \%$. El bajo contenido de materia seca en los forrajes se ha considerado como un 
factor que reduce el consumo voluntario en los rumiantes, especialmente cuando el contenido de materia seca es menor a 25\% (Pasha et al., 1994), ya que el alto contenido de agua puede ocasionar un llenado físico a nivel ruminal (Forbes, 1995). En el presente estudio, esa diferencia se pudo constatar, ya que los tres tratamientos que presentaron un contenido de materia seca cercano o superior al $25 \%$, mostraron los mayores consumos de materia seca.

Al comparar el consumo de materia seca obtenido en los tratamientos de estrella africana con y sin alimento (930 y 780 g.animal ${ }^{-1} . d^{-1}$, respectivamente) y forraje de maíz con alimento (800 g.animal ${ }^{-1} \cdot \mathrm{d}^{-1}$ ), con valores de consumo reportados en otros ensayos llevados a cabo en Costa Rica, donde se emplearon gramíneas, puede notarse como los valores obtenidos en el presente ensayo superan a los obtenidos por otros autores. Así por ejemplo, Elizondo (2004ab, 2005) evaluó el consumo en ensayos con jaulas individuales, donde se emplearon cabras secas de la raza LaMancha con pesos promedios de $38 \mathrm{~kg}$, alimentadas con sorgo negro forrajero (Sorghum almun). En dichas pruebas el consumo de materia seca rondó los 350 g.animal ${ }^{-1} \cdot d^{-1}$.

Vargas (2009) por su parte, realizó un trabajo donde evaluó el consumo de pasto prodigioso (Trypsacum laxum) para medir el impacto del nivel de ofrecimiento sobre el consumo. La gramínea se ofreció sin ningún proceso de picado en tres cantidades crecientes representadas como porcentaje del peso corporal $(7,5 ; 10,0$ y $12,5 \%)$ a cabras secas LaMancha, con $40 \mathrm{~kg}$ de peso vivo, obteniendo 342,03 gramos de MS.animal ${ }^{-1} \cdot$ día $^{-1}$ como consumo promedio para los tres niveles de ofrecimiento.

En los últimos trabajos realizados en Costa Rica para evaluar el consumo en cabras, se consideró suplementar la dieta forrajera con alimentos balanceados, para cubrir los requerimientos que el forraje no satisface y reducir así desbalances nutricionales que afectarán negativamente el consumo. En este sentido, Chacón (2008) evaluó el consumo de forraje de king grass suplementado con $1,0 \mathrm{~kg}$ de alimento balanceado.animal ${ }^{-1} \cdot \mathrm{d}^{-1}$. Este autor evaluó el consumo del forraje comparando tres edades de corte de 60, 75 y 90 días en cabras secas LaMancha, Toggemburg y Saanen, con un peso promedio de $44 \mathrm{~kg}$. En este experimento, el consumo de forraje solamente llegó a ser en promedio de $250,92 \mathrm{~g}$ de $\mathrm{MS}$.animal ${ }^{-1} \cdot \mathrm{d}^{-1}$.

Herrera et al. (2009) evaluaron el consumo y la variación en la composición de la leche de cabras LaMancha con un peso promedio de $50 \mathrm{~kg}$, alimentadas con 1,0 kg de alimento balanceado $y$ tres diferentes forrajes (estrella, sorgo negro forrajero $y$ morera). El consumo de forraje correspondiente fue de 760, 690 y $630 \mathrm{~g}_{\text {.animal }}{ }^{-1} \cdot \mathrm{d}^{-1}$. A 
pesar de que este último trabajo presentó un consumo mayor de materia seca, es probable que éste aumento estuviera influenciado por el estado fisiológico de la lactancia en los animales y no por la suplementación con alimento balanceado.

Elizondo (2004a) evaluó el consumo de morera en cabras secas de la raza LaMancha con pesos de $38 \mathrm{~kg}$, y obtuvo un consumo promedio de 737,32 $\mathrm{g}_{\text {de }} \mathrm{MS}$.animal ${ }^{-1} \cdot \mathrm{d}^{-1}$.

Continuando con este tipo de ensayos, Rodríguez y Elizondo (2012) al evaluar la calidad, selección, consumo y digestibilidad aparente de morera y estrella africana en cabras ofrecidos de forma fresca y parcialmente deshidratados, determinaron consumos desde 760 hasta $1120 \mathrm{~g}$ de $\mathrm{MS}$ animal ${ }^{-1} \cdot \mathrm{d}^{-1}$, lo que correspondió a consumos de 2,09 a $3,17 \%$ del peso vivo, respectivamente.

El consumo de materia seca con relación al peso vivo varió significativamente entre tratamientos $(P<0,05)$, el forraje de estrella africana con alimento balanceado se consumió en una relación del 1,63\% del peso vivo, mientras que para el forraje de maíz sin alimento balanceado la relación fue de 0,86\%.

\section{Análisis de la composición química de las dietas.}

En el Cuadro 2 se aprecia el consumo y composición bromatológica de las dietas ofrecidas y consumidas. Al analizar la composición química de la dieta ofrecida, puede observarse como, de manera general, se obtuvieron diferencias estadísticas significativas $(P<0,05)$ entre los diferentes tratamientos.

El porcentaje de proteína cruda ofrecida y consumida tuvo diferencias significativas $(P<0,05)$ entre tratamientos. Puede notarse como el porcentaje de proteína cruda en el material ofrecido y consumido fue siempre superior en la dieta de forraje de maíz, no obstante debido al bajo consumo de materia seca en la dieta de forraje de maíz sin alimento balanceado, la cantidad de proteína cruda consumida (g.animal-1.d-1) no superó los 70 gramos. El mayor consumo de proteína cruda fue de 130 gramos para la dieta de forraje de maíz con alimento. Rodríguez y Elizondo (2012) reportaron consumos de proteína cruda que oscilaron entre 75 y 135 g.animal-1.d-1.

La concentración de proteína cruda encontrada en el forraje de maíz es muy similar a la reportada por Elizondo y Boschini (2001) y Elizondo (2011), y la concentración en el forraje de estrella africana es inferior a la reportada por Villalobos et al. (2013) en la zona de Monteverde que fue de $14,23 \%$ a los 28 días de rebrote. 
Cuadro 2. Consumo y composición química de las dietas ofrecidas en base seca.

\begin{tabular}{lccccc}
\hline & \multicolumn{2}{c}{ Forraje de maíz } & & \multicolumn{2}{c}{ Forraje de estrella africana } \\
\cline { 2 - 3 } \cline { 6 - 7 } Dieta ofrecida & Sin alimento & Con alimento & & Sin alimento & Con alimento \\
\hline Proteína cruda & & & & & \\
Ofrecida, \% & $13,92^{\mathrm{b}}$ & $15,97^{\mathrm{a}}$ & & $11,14^{\mathrm{c}}$ & $11,57^{\mathrm{c}}$ \\
Consumida, \% & $13,45^{\mathrm{a}}$ & $13,92^{\mathrm{a}}$ & & $12,31^{\mathrm{b}}$ & $11,11^{\mathrm{c}}$ \\
Consumida, g/d & $0,07^{\mathrm{c}}$ & $0,13^{\mathrm{a}}$ & & $0,10^{\mathrm{b}}$ & $0,11^{\mathrm{b}}$
\end{tabular}

Fibra detergente neutro (FDN)

$\begin{array}{lrrrr}\text { Ofrecida, \% } & 66,98^{\mathrm{c}} & 44,70^{\mathrm{a}} & 71,04^{\mathrm{d}} & 60,15^{\mathrm{b}} \\ \text { Consumida, \% } & 59,54^{\mathrm{b}} & 42,86^{\mathrm{a}} & 69,81^{\mathrm{c}} & 61,27^{\mathrm{b}} \\ \text { Consumida, g/d } & 0,30^{\mathrm{a}} & 0,35^{\mathrm{a}} & 0,51^{\mathrm{b}} & 0,56^{\mathrm{b}}\end{array}$

Fibra detergente ácido (FDA)

$\begin{array}{lcccc}\text { Ofrecida, \% } & 44,24^{\mathrm{b}} & 25,43^{\mathrm{a}} & 40,77^{\mathrm{b}} & 29,52^{\mathrm{a}} \\ \text { Consumida, \% } & 39,42^{\mathrm{b}} & 26,53^{\mathrm{a}} & 38,59^{\mathrm{b}} & 29,22^{\mathrm{a}} \\ \text { Consumida, g/d } & 0,20 & 0,25 & 0,30 & 0,28\end{array}$

\section{Cenizas}

$\begin{array}{lcrrr}\text { Ofrecida, \% } & 10,25^{\mathrm{b}} & 7,50^{\mathrm{a}} & 10,73^{\mathrm{b}} & 8,55^{\mathrm{a}} \\ \text { Consumida, \% } & 9,06^{\mathrm{b}} & 7,32^{\mathrm{a}} & 10,85^{\mathrm{c}} & 9,00^{\mathrm{b}} \\ \text { Consumida, g/d } & 0,04^{\mathrm{a}} & 0,06^{\mathrm{ab}} & 0,08^{\mathrm{b}} & 0,09^{\mathrm{b}}\end{array}$

\section{Celulosa}

$\begin{array}{lcccc}\text { Ofrecida, \% } & 37,43^{\mathrm{b}} & 20,09^{\mathrm{a}} & 34,73^{\mathrm{b}} & 25,90^{\mathrm{a}} \\ \text { Consumida, \% } & 33,98^{\mathrm{b}} & 22,85^{\mathrm{a}} & 33,09^{\mathrm{b}} & 26,85^{\mathrm{a}} \\ \text { Consumida, g/d } & 0,20 & 0,21 & 0,25 & 0,25\end{array}$

Hemicelulosa

$\begin{array}{lcrrr}\text { Ofrecida, \% } & 26,07^{\mathrm{b}} & 19,27^{\mathrm{a}} & 30,27^{\mathrm{b}} & 30,63^{\mathrm{b}} \\ \text { Consumida, \% } & 24,74^{\mathrm{ab}} & 16,33^{\mathrm{a}} & 31,23^{\mathrm{b}} & 31,75^{\mathrm{b}} \\ \text { Consumida, g/d } & 0,13^{\mathrm{a}} & 0,14^{\mathrm{a}} & 0,24^{\mathrm{b}} & 0,30^{\mathrm{b}}\end{array}$

\section{Lignina}

$\begin{array}{lcccc}\text { Ofrecida, \% } & 6,81^{\mathrm{b}} & 3,34^{\mathrm{a}} & 6,04^{\mathrm{b}} & 3,62^{\mathrm{a}} \\ \text { Consumida, \% } & 5,45^{\mathrm{b}} & 3,68^{\mathrm{a}} & 6,00^{\mathrm{b}} & 3,45^{\mathrm{a}} \\ \text { Consumida, g/d } & 0,03 & 0,03 & 0,05 & 0,03 \\ \text { Valores con superíndices no comunes en la misma fila difieren a } P<0,05 .\end{array}$

De acuerdo con Elizondo (2002), los requerimientos de proteína cruda para mantenimiento son de 58,1 g.animal ${ }^{-1} \cdot \mathrm{d}^{-1}$. Esto quiere decir que con base en los 
resultados obtenidos, todos los tratamientos utilizados en este ensayo superaron dicho requerimiento, por lo que los animales dispusieron de un exceso de proteína cruda que podría ser utilizado para llenar los requerimientos de otras necesidades fisiológicas.

La concentración de FDN en la materia seca ofrecida y consumida difirió significativamente $(P<0,05)$ entre las dietas estudiadas. A pesar de ello, las cabras en el tratamiento de forraje de maíz consumieron en promedio la menor cantidad de FDN (325 g), en tanto que las cabras en el tratamiento de estrella fresca, consumieron la mayor cantidad (535 g). Es importante observar como los valores de pared celular disminuyen claramente debido a la utilización de alimento balanceado.

La concentración de FDA en la materia seca de las dietas, mostró un comportamiento esperado. Mientras no se presentaron diferencias en el consumo de este nutriente en las dietas ofrecidas, la concentración en las dietas ofrecidas y consumidas mostró diferencias significativas $(P<0,05)$, siendo estos valores inferiores en las dietas donde se ofreció alimento balanceado. El consumo de este nutriente fue en promedio de 257 g.animal ${ }^{-1} \cdot \mathrm{d}^{-1}$.

El porcentaje de cenizas en las dietas fue diferente entre tratamientos $(P<0,05)$ y el menor consumo lo presentaron los animales a los que se les ofreció el forraje de maíz sin alimento balanceado (40 g.animal ${ }^{-1}$ ). El porcentaje de celulosa en las dietas ofrecidas osciló entre 20,09 y $37,43 \%$, en tanto que la concentración de hemicelulosa varió entre 19,27 y 30,63\%. El contenido de lignina en el material ofrecido presentó diferencias entre tratamientos $(P<0,05)$. Sin embargo, no se presentaron diferencias en cuanto al consumo diario por animal. 


\section{CONSIDERACIONES FINALES}

Tres de las cuatro dietas evaluadas en el experimento, presentaron concentraciones de materia seca superiores al $24,45 \%$.

La especie de forraje influye significativamente sobre el consumo voluntario de materia seca en cabras y el contenido de materia seca en los forrajes utilizados afectó significativamente dicho consumo.

En general, no se obtuvieron altos consumos de materia seca y los animales que consumieron forraje de estrella africana con alimento balanceado apenas alcanzaron el $1,63 \%$ de consumo con base en su peso vivo.

Las dietas ofrecidas presentaron bajas concentraciones de proteína cruda; sin embargo, con las cuatro dietas experimentales los animales lograron satisfacer sus requerimientos de proteína cruda para mantenimiento.

Como consecuencia del amplio rango de valores reportados en la literatura en lo referente a consumos de materia seca y debido a la inconsistencia de valores obtenidos en los estudios realizados en nuestro país, es importante llevar a cabo más ensayos que permitan cuantificar de manera más adecuada y precisa el consumo de nutrimentos por parte de las cabras. 
Aldrich, S., Leng, E. 1974. Producción moderna de maíz. Editorial Hemisferio Sur. Argentina. p 308.

A.O.A.C. (Association of Official Analysis Chemistry). 1980. Methods of analysis. 13th ed. Washington D.C. U.S.A. p 168.

Bernal, J. 1991. Pastos y forrajes tropicales: Producción y manejo. 2da edición. Bogotá, Colombia, Banco Ganadero. p 544.

Castro, A. 1989. Producción de leche de cabras alimentadas con (Pennisetum purpureum $\times$ P. typhoides), suplementadas con diferentes niveles de poró (Erythrina poeppigiana) y de fruto de plátano (Musa sp. Cv. 'Pelipita'). Tesis para optar por el grado de Mag. Sc. en Ciencias Agrícolas y Recursos Naturales. CATIE. Turrialba, Costa Rica. p 53.

Chacón, P. 2008. Estimación del aprovechamiento en ganado caprino del Pennisetum purpureum cv. King Grass cosechado a tres diferentes edades de rebrote. Tesis para optar por el título de Licenciado en Ingeniería Agronómica con énfasis en Zootecnia. Universidad de Costa Rica. San José, Costa Rica. p 135.

Cháves, C. 2005. Calidad y consumo de mezclas de Cratylia argentea y Sorgo Forrajero (Sorghum bicolor (L.) Moench) con y sin melaza, ensiladas en bolsas plásticas. Tesis para optar por el título de Licenciado en Ingeniería Agronómica con énfasis en Zootecnia. Universidad de Costa Rica. San José, Costa Rica. p 56.

Elizondo, J. 2002. Estimación lineal de los requerimientos nutricionales del NRC para cabras. Agronomía Mesoamericana. 13(2):159-163.

Elizondo, J. 2004a. Calidad nutricional y consumo de morera (Morus alba), ramio (Bohemeria nivea (L) GAUD) y sorgo negro forrajero (Sorghum almum) en cabras. Agronomía Mesoamericana. 15(2):209-213. 
Elizondo, J. 2004b. Consumo de sorgo negro forrajero (Sorghum almum) en cabras. Agronomía Mesoamericana. 15(1):77-80.

Elizondo, J. 2005. Calidad y consumo de sorgo negro forrajero (Sorghum almum), ramio (Bohemeria nivea (L) Gaud) y mezcla de ambos. Pastos y Forrajes. 28(3):247-252.

Elizondo, J. 2008. El pasto estrella africana: características nutricionales y aspectos de manejo. Ventana Lechera. 10:26-27.

Elizondo, J. 2011. Influencia de la variedad y altura de cosecha sobre el rendimiento y valor nutritivo de maíz para ensilaje. Agronomía Costarricense. 35(2):105-111.

Elizondo, J., Boschini, C. 2001. Efecto de la densidad de siembra sobre el rendimiento y calidad del forraje de maíz. Agronomía Mesoamericana. 12(2):181-187.

Forbes, J. 1995. Ruminant gastrointestinal tract. In: Voluntary food intake and diet selection in farm animals. Forbes, J. (Ed). CAB International, Wallingford. p 5980.

Fuentes, J., Cruz, A., Castro, L., Gloria, G., Rodríguez, S., Ortiz, B. 2001. Evaluación de variedades e híbridos de maíz (Zea mays L.) para ensilado. Agronomía Mesoamericana. 12(2):193-197.

Goering, H., Van Soest, P. 1970. Forage fiber analysis (Apparatus, reagents, procedures and some applications). Agricultural Handbook № 379. ARSUSDA,Washington, D.C. p 76.

Herrera, H., Vargas, F., Boschini, C., Chacón, A. 2009. Variación bromatológica de la leche de cabras LaMancha alimentadas con diferentes forrajes. Agronomía Mesoamericana. 20(2):381-390.

Hristov, A., Price, W., Shafii, B. 2004. A meta analysis examining the relationship among dietary factors, dry matter intake and milk protein yield in dairy cows. J. Dairy Sci. 87: 3052-3062. 
McDonald, P., Edwards, R.A., Greenhalgh, J.F., Morgan, C.A. 2002. Animal Nutrition. $6^{\text {th }}$ edition. Prentice Hall. p 693.

Pasha, T., Priegge, E., Russell, R., Bryan, W. 1994. Influence of moisture content of forage diets on intake and digestion by sheep. Journal of Animal Science. 72: 2455-2463.

Rodríguez, J., Elizondo, J. Consumo, calidad nutricional y digestibilidad aparente de morera (Morus alba) y pasto estrella (Cynodon nlemfuensis) en cabras. Agronomía Costarricense. 36(1):13-23.

Rodríguez, Z. 1989. Producción de leche de cabras alimentadas con dos especies forrajeras arbóreas: poró (Erythrina poeppigiana) y madero negro (Gliricidia sepium) suplementadas con plátano pelipita (Musa sp. Cv. 'Pelipita'). Tesis para optar por el título de Licenciada en Ingeniería Agronómica con énfasis en Zootecnia. Universidad de Costa Rica. San José, Costa Rica. p 75.

SAS (SAS Institute Inc, US). 2004. SAS/STAT 9.1 User`s guide. Version 9.1 Cary, N.C. p 5121.

Samur, C. 1984. Producción de leche de cabras alimentadas con King grass (Pennisetum purpureum) y poró (Erythrina poeppigiana), suplementadas con fruto de banano (Musa sp. Cv. 'Cavendish'). Tesis para optar por el grado de Mag. Sc. en Ciencias Agrícolas y Recursos Naturales. CATIE. Turrialba, Costa Rica. P 51.

Seleka, T.B. (2001) Determinants of short-run supply of small ruminants in Botswana. Small Ruminant Research. 40: 203-214.

Skerman, P. 1992. Gramíneas tropicales. Roma. FAO. p 849.

Vallejo, M.A. 1995. Efecto del premarchitado y la adición de melaza sobre la calidad del ensilaje de diferentes follajes de árboles y arbustos tropicales. Tesis para optar por el título de Magister Scientiae. Centro Agronómico Tropical de Investigación y Enseñanza. Turrialba. Costa Rica. p 115. 
Vargas, F. 2009. Consumo y calidad del forraje Trypsacum laxum de un año de edad en cabras. Agronomía Mesoamericana. 20(2):391-398.

Villalobos, L., Arce, J., WingChing, R. 2013. Producción de biomasa y costos de producción de pastos estrella africana (Cynodon nlemfuensis), kikuyo (Kikuyuocloa clandestina) y ryegrass perenne (Lolium perenne) en lecherías de Costa Rica. Agronomía Costarricense. 37(2):91-103. 